\title{
Near-Field Beamforming Array for Detecting Elastic Waves in the Earth
}

\author{
Seung-Ho Lee and Waymond R. Scott, Jr. \\ School of Electrical and Computer Engineering \\ Georgia Institute of Technology \\ Atlanta, GA 30332-0250
}

\begin{abstract}
A near-field beamforming array is investigated for use in a radar system that is part of a hybrid elastic/electromagnetic technique for detecting land mines. The radar is used to measure the displacement of the surface of the earth and land mines due to elastic waves in the earth. The beamforming array is used to obtain a sufficiently small spatial resolution for the measurement of the displacement while allowing an adequate standoff distance for the radar. Both theoretical and experimental models are developed to investigate the viability of the beamforming array.
\end{abstract}

Keywords: Beamforming Array, Mine Detection, Displacement Measurement

\section{INTRODUCTION}

This work is part of a project in which a land mine detection system is being investigated that simultaneously uses both electromagnetic and elastic waves. ${ }^{1}$ The configuration of the system currently being investigated is shown in Fig. 1. The system consists of an electromagnetic radar and an elastic wave source. An elastic wave is launched into the earth, and the elastic wave causes the surface of the earth and the mine to be displaced. The radar is used to measure the displacements and, thus, detect the mine. Currently, the radar uses an open-ended waveguide or a small horn as the antenna. The radar can detect displacements as small as $10^{-9} \mathrm{~m}$ and has a spatial resolution approximately $2 \mathrm{~cm}$ as currently configured. However, to obtain this spatial resolution, the antenna must be placed within a few centimeters of the surface of the earth.

In this work, we investigate the use of a near-field beamforming array so that the antenna can be placed farther from the surface while maintaining sufficiently small spatial resolution. The array can be implemented either by constructing an array of $\mathrm{N}$ elements and recording the signal from all of the elements simultaneously or by using a single element that is scanned to construct a synthetic array. The most practical solution will probably be to use a combination of these techniques and scan a subset of the N-element array to more quickly construct the synthetic array.

A theoretical and an experimental model has been developed for the beamforming array. These models are used to examine the feasibility of the beamforming array. The theoretical model is based on the integral equation method. It simulates the signals received by the antenna due to the displacement of the surface, and the beamforming array is implemented to reconstruct the displacement. The experimental model consists of an electrodynamic transducer to generate elastic waves, a tank filled with damp sand to simulate the earth, simulated mines, and a radar to measure the displacements of the surface of the sand. The beamforming algorithm is shown to improve the resolution of both the theoretical and experimental results.

\section{THEORETICAL MODEL}

A schematic diagram of the theoretical model is shown in Fig. 2. The array consists of $\mathrm{I} \times \mathrm{J}$ elements that are placed at the height $\mathrm{h}$ and parallel to the $x-y$ plane. All elements are assumed to be identical. To make the model simple, the surface of the earth is modeled as a perfect electric conducting plane. The displacement of the surface is modeled as a circular bump with a diameter $D$ and height $\Delta z$. For the $i^{t h}$ and $j^{t h}$ element, let us assume that electric and magnetic current densities $\vec{J}_{s}$ and $\vec{M}_{s}$ on the aperture of the element bounded by the surface $s^{\prime}$ are known, and are located at the position $\vec{R}^{\prime}$. The position vector $\vec{R}^{\prime}$ is used to locate the $\mathrm{M} \times \mathrm{N}$ observation points on the surface

E-mail and Tel.: S.H. Lee: gt2332b@prism.gatech.edu, 404-894-3123, W. R. Scott, Jr.: wrscott@ee.gatech.edu, 404-894-3048 


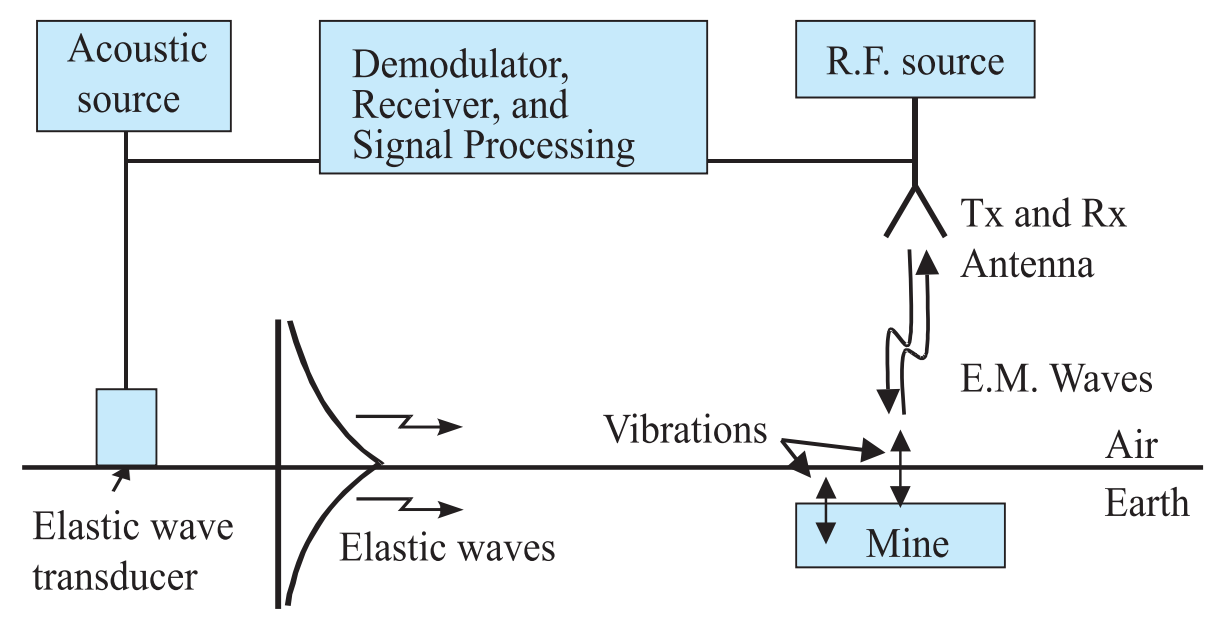

Figure 1. A system that simultaneously uses both elastic and electromagnetic waves to detect buried land mines.

of the conductor. The electric field radiated by the aperture, ignoring the effects of the conducting surface, can be expressed as $^{2}$

$$
\begin{gathered}
\vec{E}_{e, i j}^{t}(\vec{R})=\frac{-j \omega \mu_{0}}{4 \pi} \iint_{s^{\prime}}\left\{\vec{J}_{s}+\frac{1}{k_{0}}\left[-k_{0}^{2}\left(\vec{J}_{s} \cdot \hat{r}\right) \hat{r}+\frac{3}{r}\left(j k_{0}+\frac{1}{r}\right)\left(\vec{J}_{s} \cdot \hat{r}\right) \hat{r}-\frac{\vec{J}_{s}}{r}\left(j k_{0}+\frac{1}{r}\right)\right]\right\} \frac{e^{-j k_{o} r}}{r} d s^{\prime}, \\
\vec{E}_{m, i j}^{t}(\vec{R})=-\frac{1}{4 \pi} \iint_{s^{\prime}}\left[\vec{M}_{s} \times \hat{r}\left(j k_{0}+\frac{1}{r}\right)\right] \frac{e^{-j k_{o} r}}{r} d s^{\prime},
\end{gathered}
$$

where $\hat{r}=\frac{\vec{R}-\vec{R}^{\prime}}{\left|\vec{R}-\vec{R}^{\prime}\right|}$ and $r=\left|\vec{R}-\overrightarrow{R^{\prime}}\right|$. The total electric field is then

$$
\vec{E}_{i j}^{t}(\vec{R})=\vec{E}_{e, i j}^{t}(\vec{R})+\vec{E}_{m, i j}^{t}(\vec{R}) .
$$

Since the conducting surface is in the far-field of the source, the magnetic field is

$$
\vec{H}_{i j}^{t}(\vec{R})=\frac{1}{\eta_{o}} \hat{s} \times \vec{E}_{i j}^{t}(\vec{R})
$$

where $\hat{s}=\frac{\vec{R}}{|\vec{R}|}$ (radial unit vector). Next, the surface current density is obtained from the tangential components of the magnetic field on the surface. Since the surface of the conductor is approximately planar, the surface current density can be approximated by

$$
\vec{J}_{i j}^{e q}(\vec{R})=2 \hat{z} \times \vec{H}_{i j}^{t}(\vec{R}) .
$$

Let $\Delta \vec{E}_{i j}^{r}(\vec{R})$ be the electric field at the center of the $i^{t h}$ and $j^{t h}$ element due to the current element at the location $\vec{R}$. Then, the voltage received by the $i^{t h}$ and $j^{t h}$ element is given by

$$
V_{i j}^{r} \cong \frac{1}{2} \sum_{m=1}^{M} \sum_{n=1}^{N} \Delta \vec{E}_{i j}^{r}(\vec{R}) \cdot \vec{l}_{i j}^{e}(\vec{R}),
$$

where $\vec{l}_{i j}^{e}(\vec{R})$ is the vector effective height. Here the vector effective height is used to determine the voltage induced on the open-circuit terminals of the antenna from the received field. The effective height is determined from the radiated field $(3)$ of the antenna ${ }^{3}$ :

$$
\vec{l}_{i j}^{e}(\vec{R})=\frac{4 \pi|\vec{R}| \vec{E}_{i j}^{t}(\vec{R})}{-j \eta_{0} k_{0} I_{t} e^{-j k_{0}|\vec{R}|}} .
$$

For each element, the above steps must be repeated to synthesize the beamforming array. 


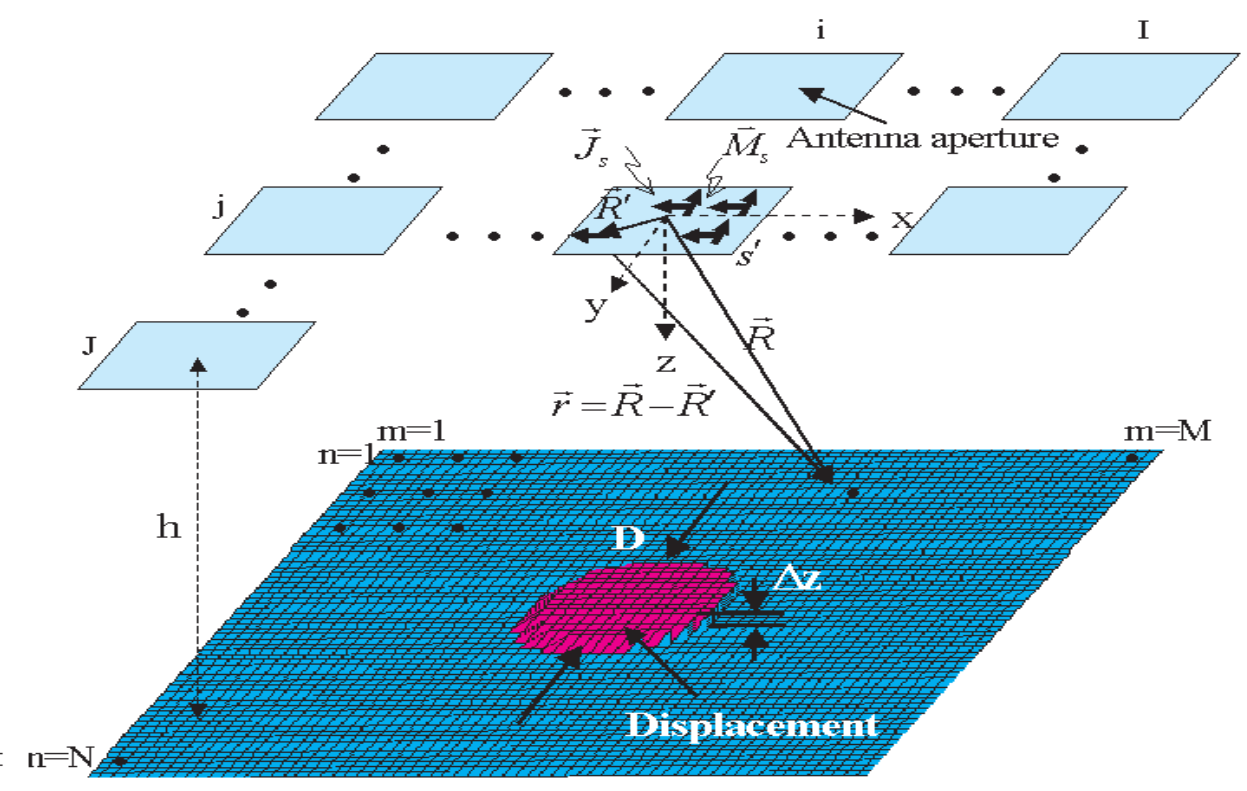

Figure 2. A schematic diagram of the theoretical model

\section{BEAMFORMING ALGORITHM}

After obtaining the received voltage at each element position, a beamforming algorithm can be applied. A schematic diagram of the beamforming array is shown in Fig. 3. The configuration of the array is the same as in Fig. 2 except for the coordinate system. The location of the element is indicated by $\vec{R}^{\prime}$, and the received voltage at each element position is known. The array is focused at an arbitrary focal point located at the position $\vec{R}_{f}$. The received voltages of the elements are summed with the appropriate weighting function to focus the array at the focal point. The phase of the weighting function is chosen so that the voltages will add constructively by using the conjugate focusing method, and the amplitude of the weighting function is chosen to control the sidelobe levels of the array. The output of the array is then

$$
V_{A}\left(\vec{R}_{f}\right)=\sum_{i=1}^{I} \sum_{j=1}^{J} V_{i j}^{r}\left(\vec{R}^{\prime}\right) W\left(\vec{R}_{f}, \vec{R}^{\prime}\right) e^{j \alpha_{i j}\left(\vec{R}_{f}\right)},
$$

where $W$ is a window function used to adjust the amplitude of the weighting function, and $\alpha_{i j}\left(\vec{R}_{f}\right)=(-1)$ \{phase of $\left.\Delta V_{i j}^{r}\left(\overrightarrow{R_{f}}\right)\right\}$, where $\Delta V_{i j}^{r}\left(\overrightarrow{R_{f}}\right)=\Delta \vec{E}_{i j}^{r}\left(\overrightarrow{R_{f}}\right) \cdot \vec{l}_{i j}^{e}\left(\overrightarrow{R_{f}}\right)$. We have determined empirically that a Gaussian window function performs well:

$$
W\left(\vec{R}_{f}, \vec{R}^{\prime}\right)=\exp \left(-\frac{1}{(0.6 \tau)^{2}}\left|\hat{z} \times\left(\overrightarrow{R_{f}}-\vec{R}^{\prime}\right)\right|^{2}\right),
$$

where $\tau$ is a parameter used to adjust the width of the window function at the $50 \%$ drop point from the peak. The window function is maximum directly above $\overrightarrow{R_{f}},\left|\hat{z} \times\left(\overrightarrow{R_{f}}-\vec{R}^{\prime}\right)\right|=0$, and it decreases with increasing distances from this point.

Parameters used in the example of the theoretical model are shown in Table 1. Using the procedure described above, the surface displacements are calculated from the outputs of the array to demonstrate the effectiveness of the beamforming array. Graphs of the reconstructed displacement of the conducting surface are shown in Fig. 4. The actual displacement and the displacements with and without beamforming are shown when the array is placed at $20 \mathrm{~cm}$ and $40 \mathrm{~cm}$ high. In these graphs, the displacements are plotted as a function of $x$ with $y=0$. Here the displacements are obtained by comparing the phase between the data obtained with and without the displacement in the model. The displacement obtained without beamforming is not a good replica of the actual displacement for either height. In order to solve this problem, the beamforming array technique was used to obtain the good image 


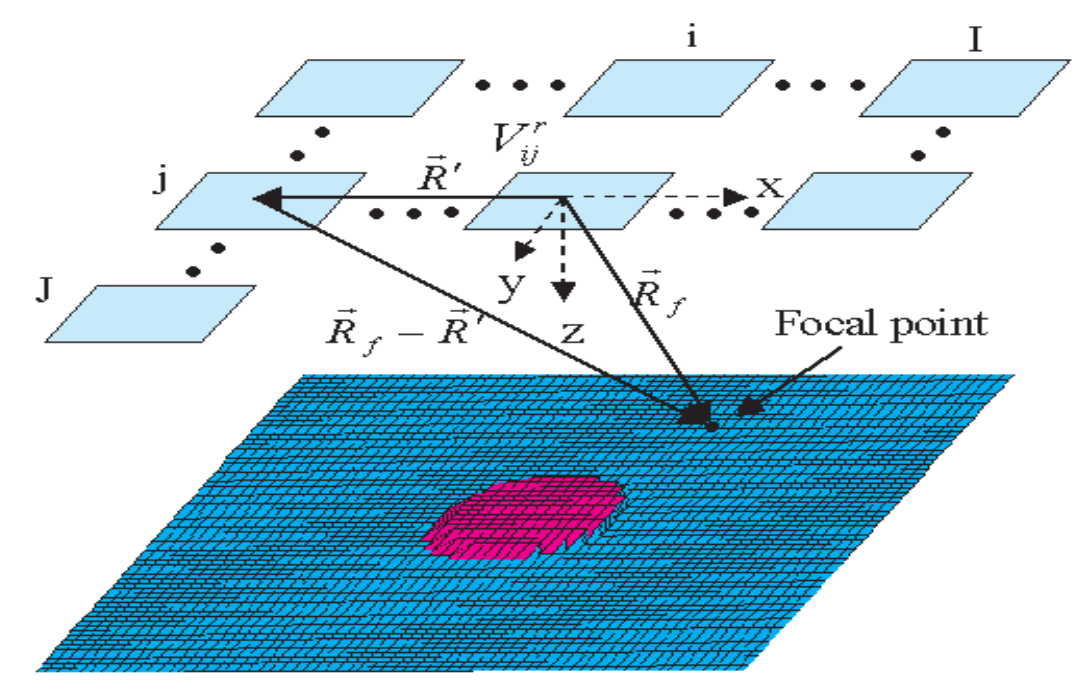

Figure 3. Beamforming array configuration

Table 1. Parameters used in the example

\begin{tabular}{|c|c|}
\hline Array & $65 \times 65$ \\
\hline Antenna & $5 \mathrm{~cm} \times 5 \mathrm{~cm}$ Pyramidal horn \\
\hline Spacing between antennas & $0.5 \lambda$ \\
\hline Frequency & $8 \mathrm{GHz}$ \\
\hline Array height, $\mathrm{h}$ & $20,40 \mathrm{~cm}$ \\
\hline Displacement & Circular with $D=20 \mathrm{~cm}$ and $\Delta z=1 \mu \mathrm{m}$ \\
\hline Plane & $1.2 \mathrm{~m} \times 1.2 \mathrm{~m}$ \\
\hline Observation points on the surface & 256 points $/ \lambda^{2}$ \\
\hline
\end{tabular}

of the displacements. The raw data were beamformed for several values of $\tau$. When the raw data are beamformed with appropriate value of $\tau$, the displacements are seen to be a better replica of the actual displacement than the results without beamforming. The spatial resolution is seen to increase with increasing $\tau$, but the sidelobe levels also increase with increasing $\tau$. Tradeoffs must be made between the spatial resolution and the sidelobe levels. ${ }^{4}$

Pseudo color graphs of the amplitude of the displacement of the surface are presented in Fig. 5 for the entire conducting region. The displacements are shown for the data obtained with and without beamforming on a 40dB scale. The sidelobes are clearly visible around the displacement in the raw data for both $\mathrm{h}=20 \mathrm{~cm}$ and $\mathrm{h}=40 \mathrm{~cm}$. The sidelobe levels increase when the height of the array increases. The top of the displacement is seen to be non-uniform in the raw data for both heights. After beamforming with the appropriate $\tau$, the sidelobes are seen to be significantly reduced, and the top of the displacement is seen to be more uniform.

\section{EXPERIMENTAL MODEL}

A radar has been designed and built to measure the displacements of the surface of the sand and the mine due to the elastic waves. ${ }^{1}$ Figure 6 is a block diagram of the radar system. The radar radiates electromagnetic waves that are reflected off of a vibrating boundary, and the reflected waves are received by the radar. A Homodyne system is used to demodulate the signals, and the signals from the I and Q channels are used to obtain the magnitude and phase of the reflected wave, which are then used to obtain the displacement of the surface. Currently, the radar is being used in a monostatic mode and uses a $5 \mathrm{~cm} \times 5 \mathrm{~cm}$ Pyramidal horn as the antenna. The radar is operated at $8 \mathrm{GHz}$ and is scanned using an $x-y$ positioner. The radar is interfaced to a personnel computer to control the positioner and to store the measured data. 


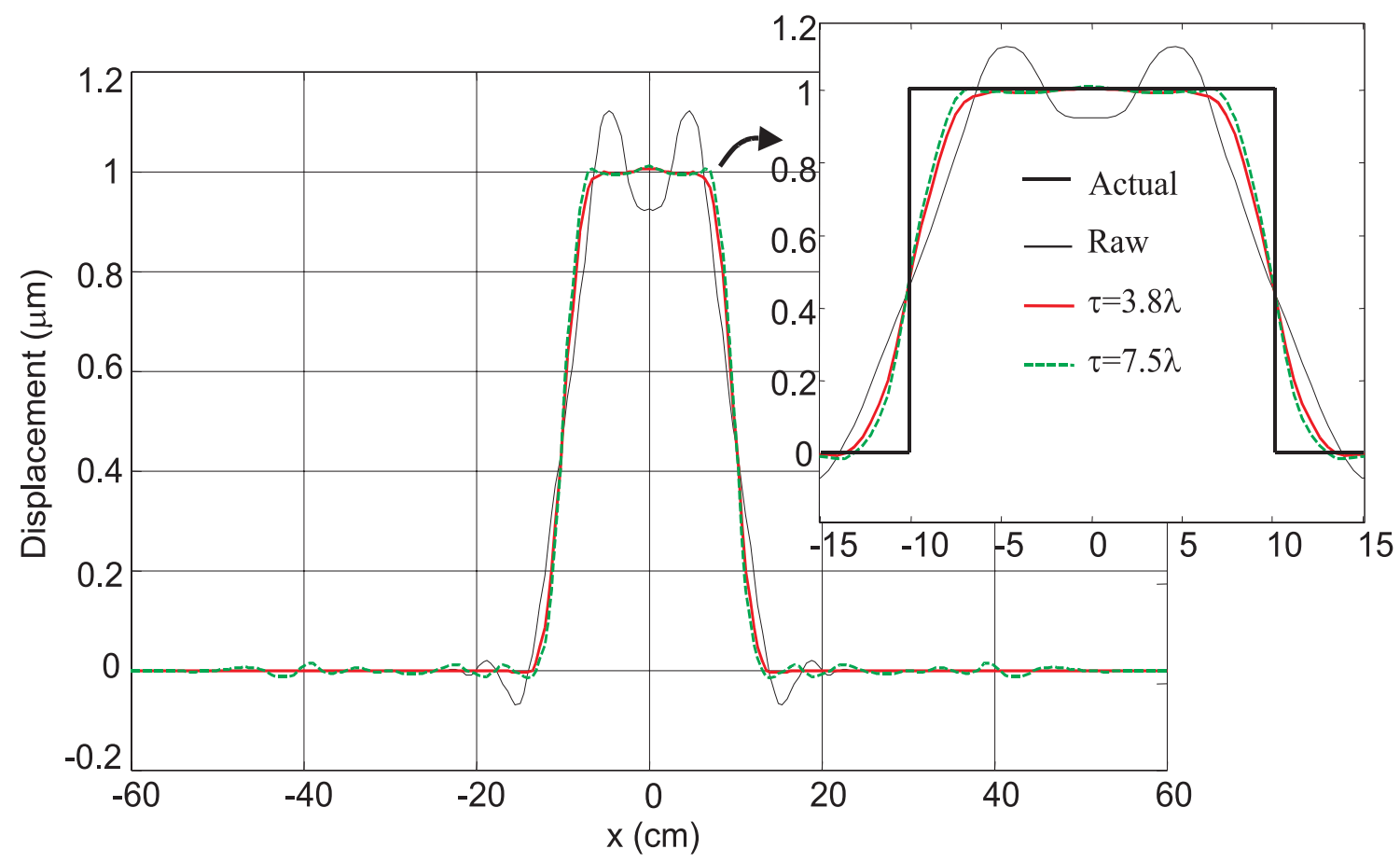

(a)

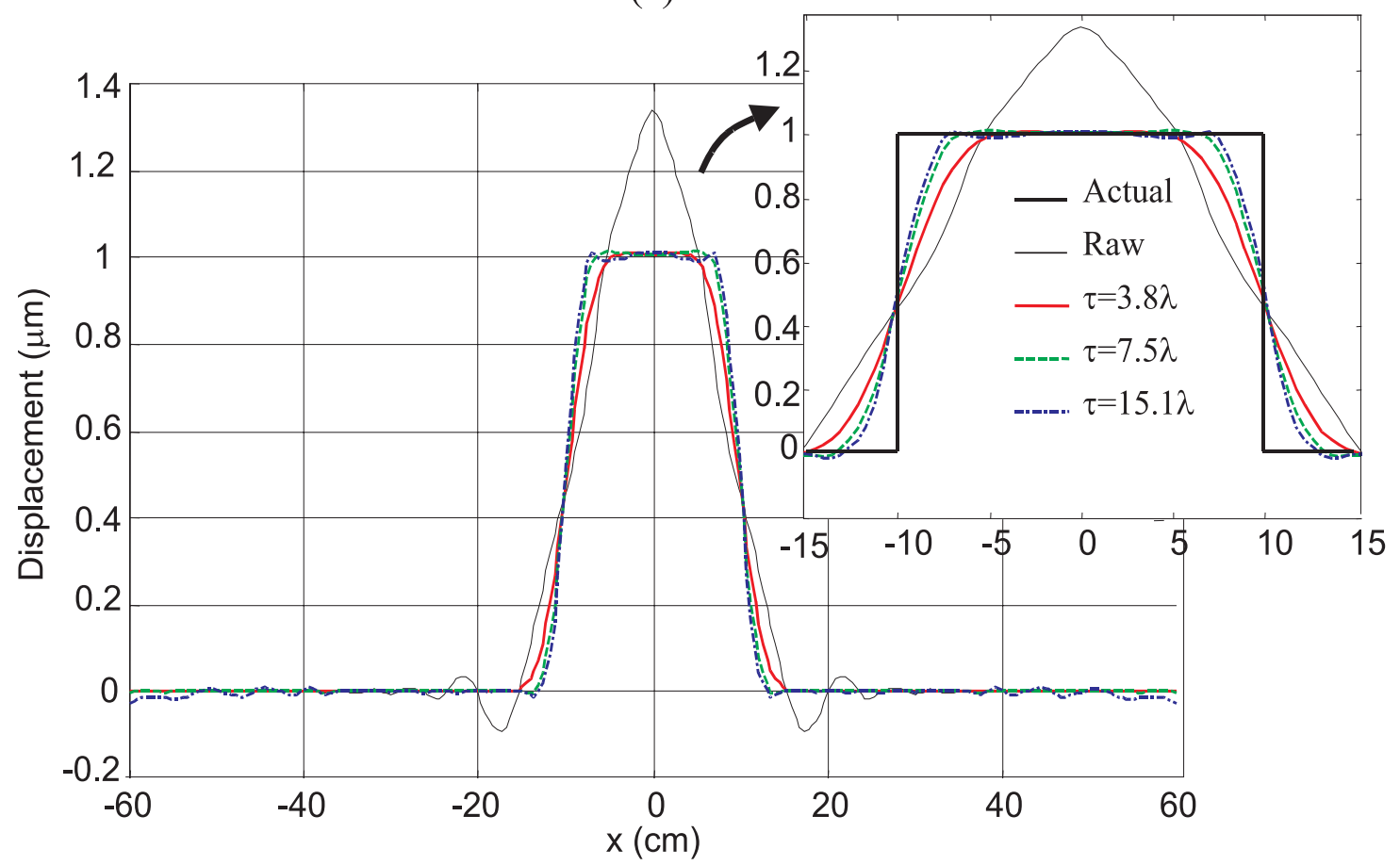

(b)

Figure 4. Reconstruction of the displacement of the surface : (a) $h=20 \mathrm{~cm}$, (b) $h=40 \mathrm{~cm}$. 


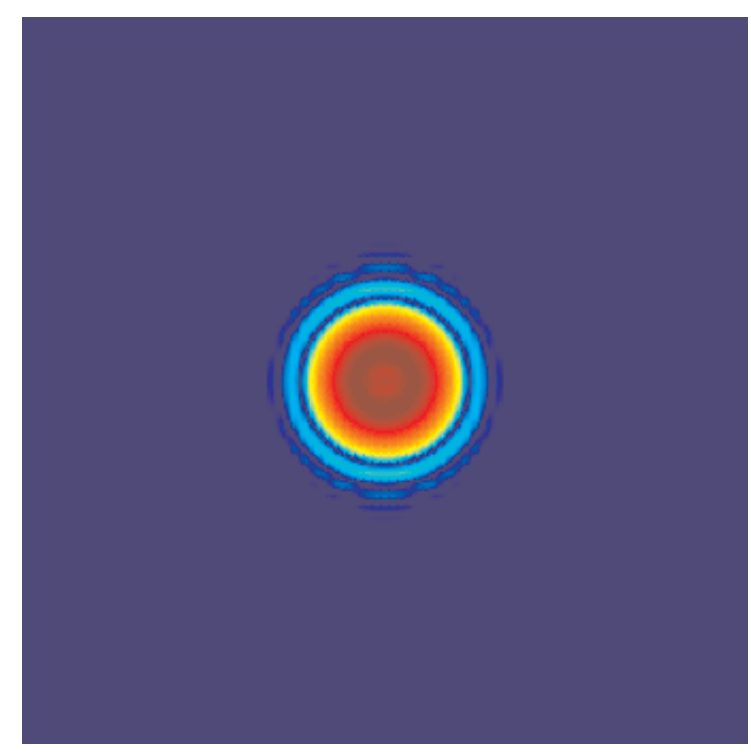

(a)

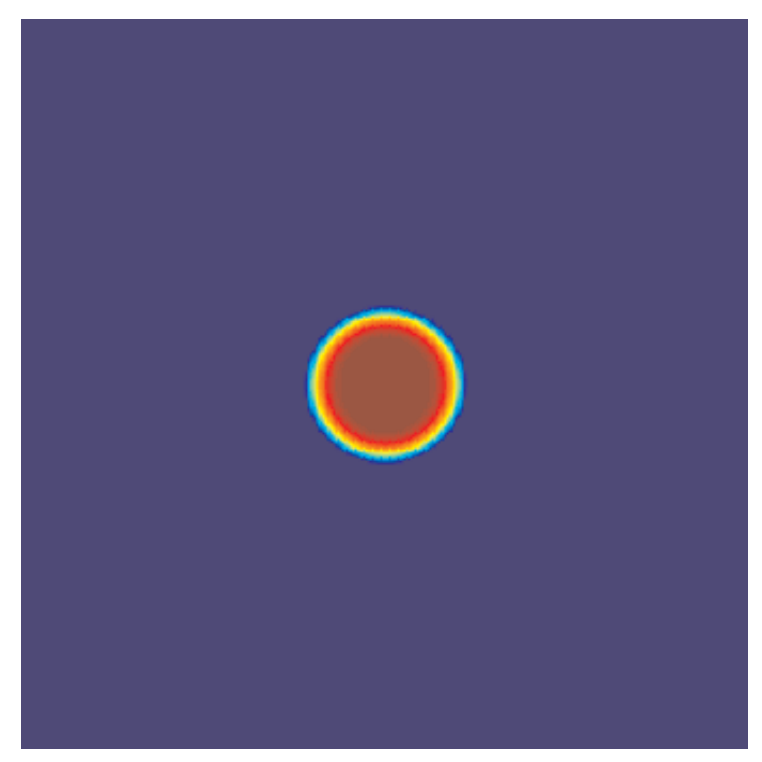

(c)

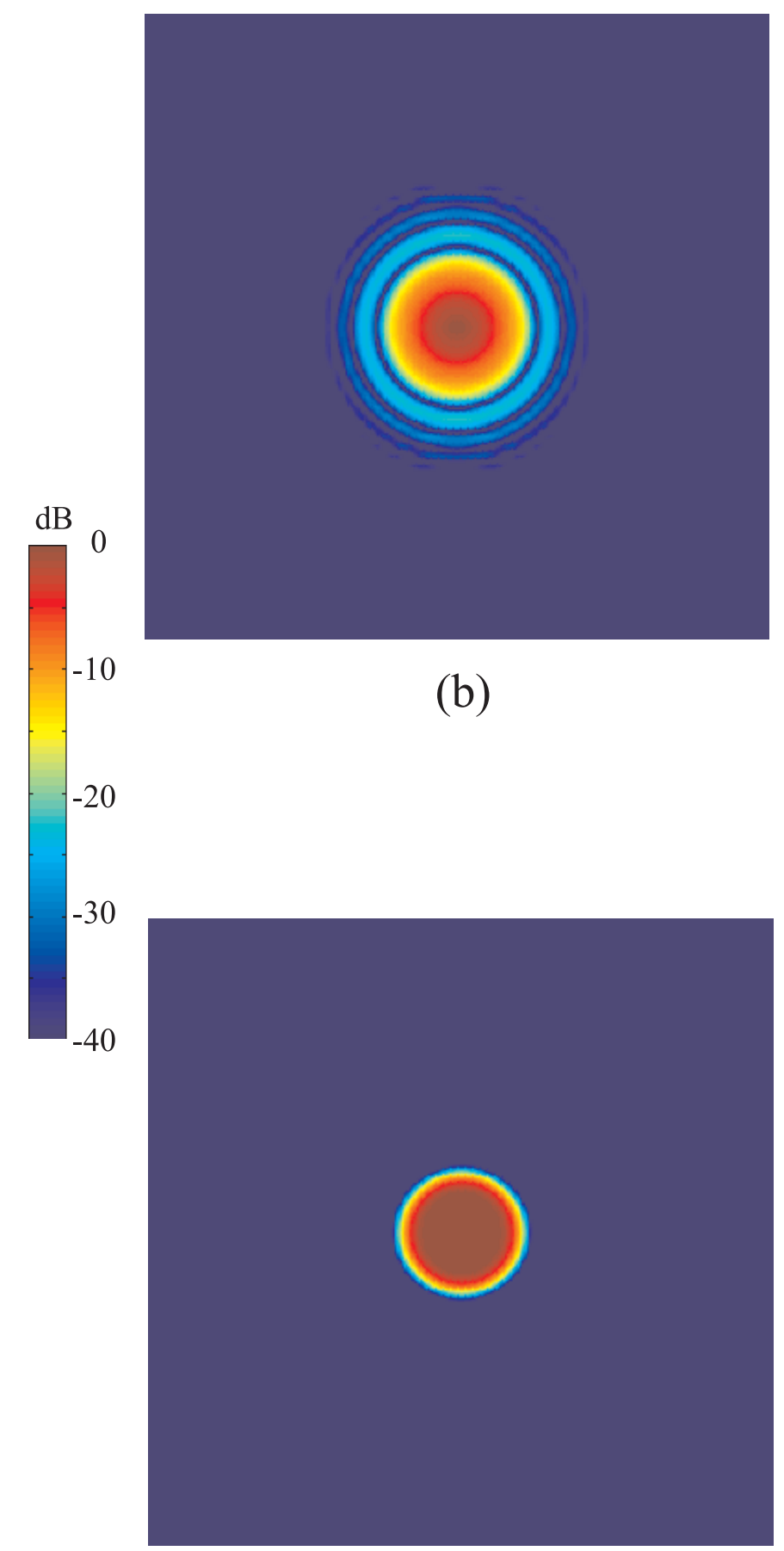

(d)

Figure 5. Pseudo color graphs of the displacement of the surface: (a) raw with $h=20 \mathrm{~cm}$, (b) raw with $\mathrm{h}=40 \mathrm{~cm}$, (c) beamformed with $\tau=3.8 \lambda$ and $\mathrm{h}=20 \mathrm{~cm}$, (d) beamformed with $\tau=7.5 \lambda$ and $\mathrm{h}=40 \mathrm{~cm}$. 


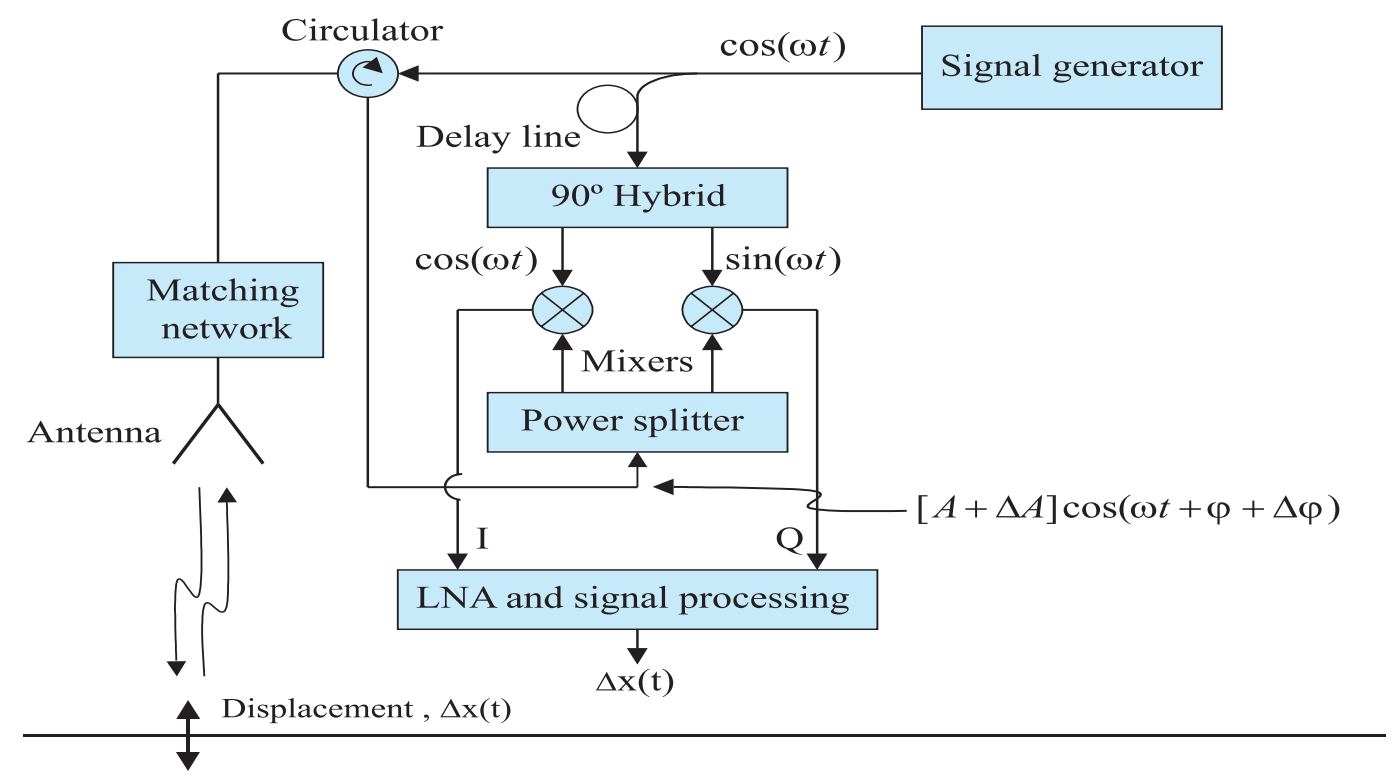

Figure 6. Radar system used in the experimental model

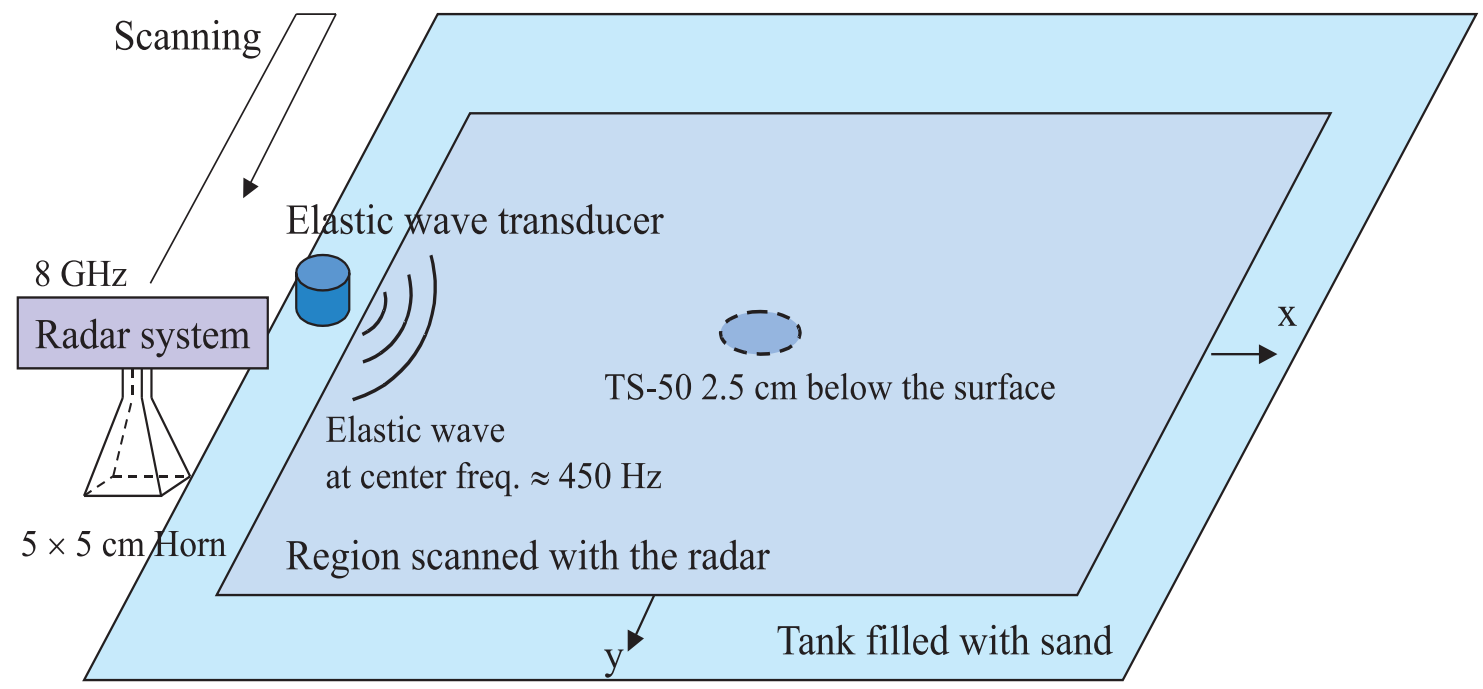

Figure 7. A schematic diagram of the experimental model

A schematic diagram of the experimental model is shown in Fig. 7. The model consists of a sandbox and a radar which is scanned over the surface of the sand to implement a synthetic beamforming array. The scanned area is 1.2 m square. A TS-50 anti-personnel mine is buried at the center of the area, and the top of the mine is $2.5 \mathrm{~cm}$ below of the surface of the sand. A transducer is placed on the surface of the sand and used to launch the elastic waves into the sand. These elastic waves travel across the surface of the sand and cause the surface of the sand and the mine to be displaced. The radar is used to measure the displacement of the surface of the sand due to the elastic waves. The response of the radar is measured on a uniform square grid of discrete positions in the scanned region. The grid consists of $65 \times 65$ equally spaced points that are spaced $1.875 \mathrm{~cm}$ apart. The displacements of the surface of the sand were measured and recorded as a function of time at each of these positions. Experiments have been performed for three different heights of the antenna. The antenna is placed at $5 \mathrm{~cm}, 20 \mathrm{~cm}$, or $40 \mathrm{~cm}$ high above the surface of the sand. 
Pseudo color graphs of the amplitude of the displacement of the surface are shown in Fig. 8 for the entire scanned region at a single instant in time. In these graphs, the elastic wave is traveling across the surface of the sand from the left hand side to the right hand side. These graphs show the displacements of the surface at a time at which the elastic wave has just passed the mine. A portion of the wave is seen to be scattered from the mine, and the displacements are seen to be biggest above the mine due to a resonance that occurs at the mine location. The resonance makes it much easier to detect the mine. ${ }^{5}$ The mine is clearly visible at the center of the scanned region in all of the graphs. The displacements are shown for the raw data and for the beamformed data with $\tau=3.8 \lambda$ and $\tau=7.5 \lambda$ for $\mathrm{h}=20 \mathrm{~cm}$ and $\mathrm{h}=40 \mathrm{~cm}$, respectively. Figures $8(\mathrm{a})-(\mathrm{c})$ show the raw measured data without beamforming for three different heights of the antenna. At a height of $5 \mathrm{~cm}$, the raw data is a good representation of the actual displacement so that we can compare this result to the other cases. The spatial resolution becomes worse as the height of the radar is increased. Therefore, the mine is seen to appear much bigger than its actual size, and some artifacts(sidelobes) are observed around the mine.

The beamforming array has been used to improve the spatial resolution for the raw measured data. The beamformed displacements are also shown in Fig. 8(d) and (e) when $\mathrm{h}=20 \mathrm{~cm}$ and $\mathrm{h}=40 \mathrm{~cm}$. The raw data was beamformed with $\tau=3.8 \lambda$ for $\mathrm{h}=20 \mathrm{~cm}$ and with $\tau=7.5 \lambda$ for $\mathrm{h}=40 \mathrm{~cm}$. In both Fig. 8(d) and (e), the results are seen to be clearly better than the results without beamforming. Since the resolution has been improved, the location and the size of the mine are more evident in these graphs. Moreover, the artifacts around the mine have been significantly reduced after beamforming.

\section{CONCLUSIONS}

The feasibility of the near-field beamforming array has been investigated using a theoretical and an experimental model. By using the beamforming array, the spatial resolution is improved so that the displacement of the surface of the earth due to the elastic waves can be measured when the antenna is placed farther from the surface. However, more work is needed to understand the limitations of the beamforming array.

\section{ACKNOWLEDGMENTS}

This work is supported in part under the OSD MURI program by the US Army Research Office under contract DAAH04-96-1-0448.

\section{REFERENCES}

1. W. R. Scott, Jr., C. Schroeder, and J. Martin, "An acousto-electromagnetic sensor for locating land mines," SPIE, AeroSense, Detection and Remediation Technologies for Mines and Minelike Targets III, pp. 176-186, April 1998.

2. S. Silver, Microwave Antenna Theory and Design, McGraw-Hill, 1949.

3. C. A. Balanis, Antenna Theory: Analysis and Design, John Wiley and Sons, 1997.

4. S. H. Lee and W. R. Scott, Jr., "Beamforming array for detecting buried land mines," SPIE, AeroSense, Detection and Remediation Technologies for Mines and Minelike Targets IV, pp. 1343-1351, April 1999.

5. W. R. Scott, Jr. and J. Martin, "Experimental investigation of the acousto-electromagnetic sensor for locating land mines," SPIE, AeroSense, Detection and Remediation Technologies for Mines and Minelike Targets IV, pp. 204-214, April 1999. 


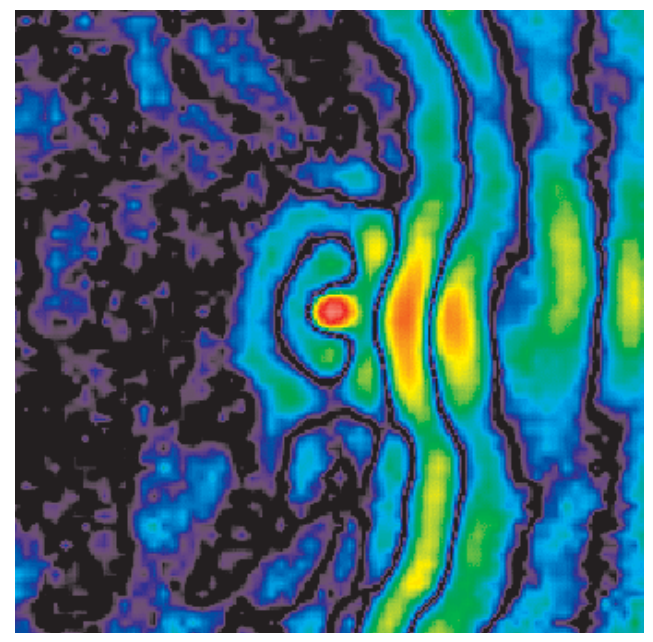

(a)

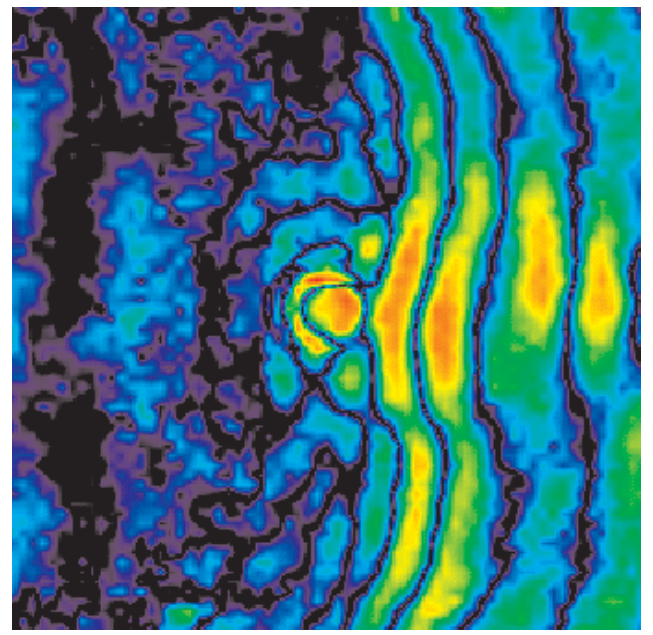

(b)

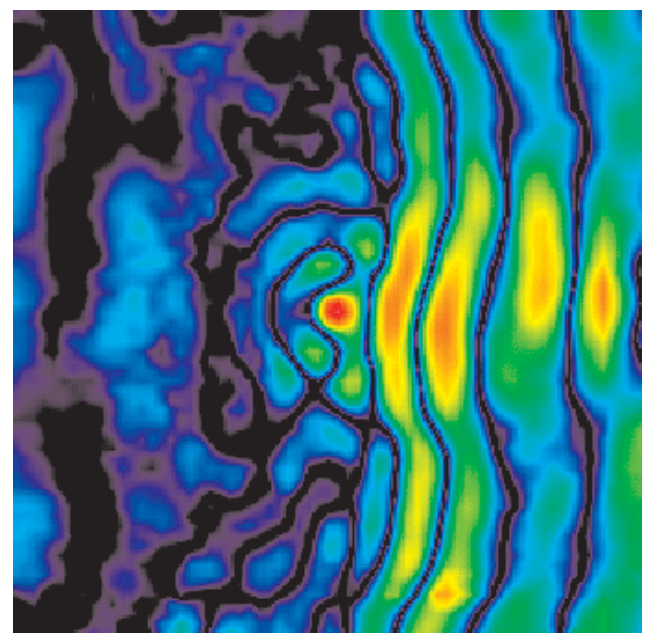

(d)

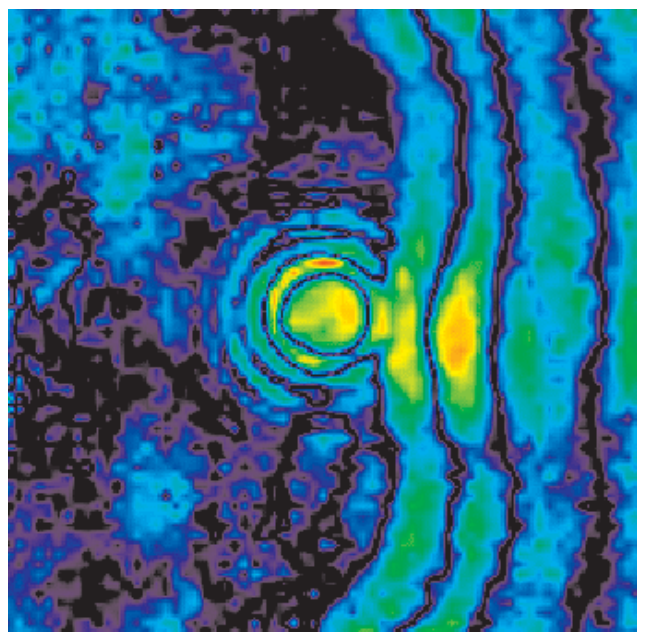

(c)

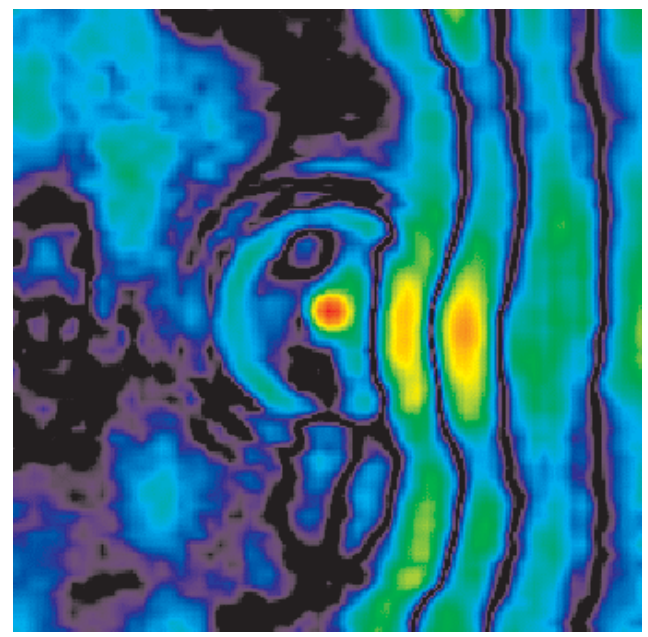

(e)

Figure 8. Pseudo color graphs of the displacement of the surface: (a) raw with $\mathrm{h}=5 \mathrm{~cm}$, (b) raw with $\mathrm{h}=20 \mathrm{~cm}$, (c) raw with $\mathrm{h}=40 \mathrm{~cm},(\mathrm{~d})$ beamformed with $\tau=3.8 \lambda$ and $\mathrm{h}=20 \mathrm{~cm}$, (e) beamformed with $\tau=7.5 \lambda$ and $\mathrm{h}=40 \mathrm{~cm}$. 